\title{
The Basis Number of the Lexicographic Product of Different Ladders with Paths and Cycles
}

\author{
Maref Yousef Mohammad Alzoubi and Reem Rafe' Ayed Al-Ta'Ani \\ Department of Mathematics, Yarmouk University, Irbid-Jordan \\ e-mail : maref@yu.edu.jo
}

Abstract. In [8] M. Y. Alzoubi and M. M. Jaradat studied the basis number of the composition of paths and cycles with Ladders, Circular ladders and Möbius ladders. Namely, they proved that the basis number of these graphs is 4 except possibly for some cases in each of them. Since the lexicographic product is noncommutative, in this paper we investigate the basis number of the lexicographic product of the different kinds of ladders with paths and cycles. In fact, we prove that the basis number of almost all of these graphs is 4.

\section{Introduction}

Throughout this paper, we consider only finite connected simple graphs. We use standard notations and terminology, and for undefined terms we refer the reader to $[10]$ and [16].

Let $G$ be a graph with $n$ vertices and $m$ edges. If we order the edges $e_{1}, e_{2}, \cdots, e_{m}$ then $G$ is associated with a vector space as follows: If $A$ is a subset of edges from $G$, then it corresponds to a $(0,1)$-vector $\left(a_{1}, a_{2}, \cdots, a_{m}\right)$ such that $a_{i}=1$ if $e_{i} \in A$, and $a_{i}=0$ if $e_{i} \notin A$. These vectors form an $m$-dimensional vector space over the field $Z_{2}$. The subspace generated by all the vectors that correspond to all the cycles of $G$ is called the cycle space of $G$, which is denoted by $\mathcal{C}(G)$. The dimension is $\operatorname{dim} \mathcal{C}(G)=m-n+1$. Usually, we say that the cycles themselves, rather than the vectors corresponding to the cycles, generate $\mathcal{C}(G)$. A basis for the cycle space $\mathcal{C}(G)$ of a graph $G$ is called a $k$ - fold if each edge of $G$ occurs in at most $k$ of the cycles in the basis. The basis number of $G$, denoted by $b(G)$, is the smallest integer $k$ such that $\mathcal{C}(G)$ has a $k$ - fold basis.

The first important result about the basis number was given in 1937 by MacLane [17] when he proved the following theorem.

Theorem 1.1(MacLane,[17]). A graph $G$ is planar if and only if $b(G) \leq 2$.

In [18] Schmeichel investigated the basis number of certain important classes of non-planar graphs, specifically, complete graphs and complete bipartite graphs.

Received December 11, 2006, and, in revised form, February 11, 2007.

2000 Mathematics Subject Classification: 05C38, 15A03.

Key words and phrases: Cycle space, The lexicographic product, Fold, Basis Number. 
Then J. Banks and E. Schemeichel [9] proved that for $n \geq 7$, the basis number of $Q_{n}$ is 4 , where $Q_{n}$ is the $n$-cube. After that, many researchers were attracted to work on finding the basis number of special classes of graphs, mainly, those obtained from different kinds of graph products. We refer interested readers to [1-8], [11-15] and their references.

The following lemmas will be used frequently in our main proofs.

Lemma 1.1(Hailat-Alzoubi[11]). Let $G$ be a graph with $p$ vertices and $q$ edges. If $|C|$ denotes the length of the cycle $C$, and $\mathcal{B}=\left\{C_{1}, C_{2}, \cdots, C_{d}:\left|C_{i}\right| \geq r\right\}$ be a $k$-fold basis of $\mathcal{C}(G)$ then $r d \leq \sum_{i=1}^{d}\left|C_{i}\right| \leq k q$, where $d=\operatorname{dim} \mathcal{C}(G)$.

Lemma 1.2(Jaradat-Alzoubi-Rawashdeh, [13]). Let $A, B$ be sets of cycles of a graph $G$, and suppose that both $A$ and $B$ are linearly independent, and $E(A) \cap E(B)$ induces a forest in $G$ (with the possibility that $E(A) \cap E(B)=\phi$ ). Then $A \cup B$ is linearly independent.

Definition 1.1. The lexicographic product (composition) of two disjoint graphs $G_{1}=\left(V_{1}, E_{1}\right)$ and $G_{2}=\left(V_{2}, E_{2}\right)$ is denoted by $G_{1}\left[G_{2}\right]$ or $G_{1} \otimes G_{2}$. It has $V(G)=$ $V_{1} \times V_{2}$ as a vertex set and its edge set is

$$
E=\left\{\left(u_{1}, v_{1}\right)\left(u_{2}, v_{2}\right) \text { either }\left[u_{1}=u_{2} \text { and } v_{1} v_{2} \in E_{2}\right] \text { or }\left[u_{1} u_{2} \in E_{1}\right]\right\} .
$$

It is worth mentioning that, in general, $G_{1}\left[G_{2}\right]$ and $G_{2}\left[G_{1}\right]$ are not isomorphic graphs since $\left|E\left(G_{1}\left[G_{2}\right]\right)\right|=p_{1} q_{2}+p_{2}^{2} q_{1}$ and $\left|E\left(G_{2}\left[G_{1}\right]\right)\right|=p_{2} q_{1}+p_{1}^{2} q_{2}$, where $\left|V\left(G_{1}\right)\right|=p_{1},\left|V\left(G_{2}\right)\right|=p_{2},\left|E\left(G_{1}\right)\right|=q_{1},\left|E\left(G_{2}\right)\right|=q_{2}$.

In [8] M. Y. Alzoubi and M. M. Jaradat studied the basis number of the composition of paths and cycles with Ladders, Circular ladders and Möbius ladders. Namely, they proved that the basis number of the graphs $P_{n}\left[L_{m}\right], P_{n}\left[C L_{m}\right], P_{n}\left[M L_{m}\right]$, $C_{n}\left[L_{m}\right], C_{n}\left[C L_{m}\right]$ and $C_{n}\left[M L_{m}\right]$ is 4 except possibly for some cases in each of them.

The purpose of this paper is to investigate the basis number of the graphs $L_{n}\left[P_{m}\right], C L_{n}\left[P_{m}\right], M L_{n}\left[P_{m}\right], L_{n}\left[C_{m}\right], C L_{n}\left[C_{m}\right]$ and $M L_{n}\left[C_{m}\right]$ taking into account that the lexicographic product is noncommutative. We use the notations $P_{n}$, $C_{n}, L_{n}, C L_{n}$ and $M L_{n}$ to denote a path, a cycle, a ladder, a circular ladder and a Möbius ladder, respectively.

\section{The Main results}

Throughout this work, $P_{n}$ and $C_{n}$ denote the path $012 \cdots(n-1)$ and the cycle $012 \cdots(n-1) 0$; respectively such that $E\left(P_{n}\right)=\{i(i+1): 0 \leq i \leq n-2\}$ and we define the edge set of $C_{n}$ by $E\left(C_{n}\right)=\{i(i+1): 0 \leq i \leq n-1\}$. The circular ladder, $C L_{n}$, will be taken as a two concentric cycles $C_{a}=a_{0} a_{1} \cdots a_{n-1} a_{0}$ and $C_{b}=b_{0} b_{1} \cdots b_{n-1} b_{0}$ with the vertex-set as follows :

$$
V\left(C L_{n}\right)=\left\{a_{0}, a_{1}, \cdots, a_{n-1}, b_{0}, b_{1}, \cdots, b_{n-1}\right\}
$$


and the edge-set as follows :

$$
E\left(C L_{n}\right)=E\left(C_{a}\right) \cup E\left(C_{b}\right) \cup\left\{a_{i} b_{i}: 0 \leq i \leq n-1\right\} .
$$

The ladder $L_{n}$ is obtained from $C L_{n}$ by deleting the edges $a_{0} a_{n-1}, b_{0} b_{n-1}$. The Möbius ladder $M L_{n}$ is obtained from $C L_{n}$ by deleting the edges $a_{0} a_{n-1}, b_{0} b_{n-1}$ and replacing them by the two edges $a_{0} b_{n-1}$ and $b_{0} a_{n-1}$.

Lemma 2.1. Let $m, n$ be two positive integers, $(n \geq 3)$, such that $4\left(m^{2}(3 n-2)+\right.$ $1) \leq 3\left(2 n m+m^{2}(3 n-2)\right)$, then $m \leq 3$.

Proof. Since $4\left(m^{2}(3 n-2)+1\right) \leq 3\left(2 n m+m^{2}(3 n-2)\right)$, we have $4 m^{2}(3 n-2)+4 \leq$ $6 n m+3 m^{2}(3 n-2)$, then $m^{2}(3 n-2)+4 \leq 6 n m$, thus $m+\frac{4}{m(3 n-2)} \leq \frac{6 n}{3 n-2} \lesseqgtr$ $\frac{6 n}{3(n-1)}$, therefore $m \lesseqgtr 4$.

Lemma 2.2. Let $m, n$ be two positive integers; $(n \geq 3)$, and $s \leq 3 m(2 n)$, such that $s+\left[\frac{6 n m+9 n m^{2}-6 m^{2}-3 s}{4}\right] \geq 3 n m^{2}-2 m^{2}+1$, where $[x]$ is the greatest integer less than or equal to $x$. Then $m \lesseqgtr 6$.

Proof. Since $\left[\frac{6 n m+9 n m^{2}-6 m^{2}-3 s}{4}\right] \leq \frac{6 n m+9 n m^{2}-6 m^{2}-3 s}{4}$ we have $3 n m^{2}-2 m^{2}+1 \leq s+\frac{6 n m+9 n m^{2}-6 m^{2}-3 s}{4}$. If we multiply this inequality by 4 and rearrange the terms we get $m^{2}(3 n-2)+4 \leq 12 n m$. If we divide the inequality by $m(3 n-2)$ we have $m+\frac{4}{m(3 n-2)} \leq 4\left(\frac{3 n}{3 n-2}\right)$. Since $\frac{3 n}{3 n-2}$ is a decreasing sequence for all $n \geq 3$ and $\frac{9}{7}$ is its maximum we have $m \lesseqgtr 6$.

It is clear that $\left|V\left(L_{n}\left[C_{m}\right]\right)\right|=2 n m,\left|E\left(L_{n}\left[C_{m}\right]\right)\right|=2 n m+m^{2}(3 n-2)$, and so $\operatorname{dim} \mathcal{C}\left(L_{n}\left[C_{m}\right]\right)=\left|E\left(L_{n}\left[C_{m}\right]\right)\right|-\left|V\left(L_{n}\left[C_{m}\right]\right)\right|+1=m^{2}(3 n-2)+1$.

Theorem 2.1. If $n \geq 3$ and $m \geq 5$, then $3 \leq b\left(L_{n}\left[C_{m}\right]\right) \leq 4$. Moreover, $b\left(L_{n}\left[C_{m}\right] t\right)=4$ for all $n \geq 3, m \geq 6$.

Proof. The graph $L_{n}\left[C_{m}\right]=C_{2 n}\left[C_{m}\right] \bigcup\left(\bigcup_{i=1}^{n-2} K_{\left(a_{i}, m\right),\left(b_{i}, m\right)}\right)$, where

$$
C_{2 n}=a_{0} a_{1} \cdots a_{n-1} b_{n-1} b_{n-2} \cdots b_{1} b_{0} a_{0} .
$$

Since $C_{2 n}\left[C_{m}\right]$ is a nonplanar subgraph of $L_{n}\left[C_{m}\right]$, the graph $L_{n}\left[C_{m}\right]$ is a nonplanar graph and thus by Maclane's theorem $b\left(L_{n}\left[C_{m}\right]\right) \geq 3$.

To prove that $b\left(L_{n}\left[C_{m}\right]\right) \leq 4$, we exhibit a $4-$ fold basis for $\mathcal{C}\left(L_{n}\left[C_{m}\right]\right)$. For each $1 \leq r \leq n-2$, define the following sets of cycles in $\mathcal{C}\left(L_{n}\left[C_{m}\right]\right)$ :

$$
A_{r}=B\left(K_{\left(a_{r}, m\right)\left(b_{r}, m\right)}\right),
$$




$$
\begin{aligned}
A_{r}^{\prime}= & \left\{\left(a_{r}, 0\right)\left(b_{r}, i\right)\left(b_{r}, i+1\right)\left(a_{r}, 0\right): 0 \leq i \leq m-2\right\} \\
& \cup\left\{\left(b_{r}, m-1\right)\left(a_{r}, i\right)\left(a_{r}, b_{r}\right)\left(b_{r}, m-1\right): 0 \leq i \leq m-2\right\}, \\
Q= & \left\{Q_{i}=\left(a_{i}, 0\right)\left(a_{i+1}, 0\right)\left(b_{i+1}, 0\right)\left(b_{i}, 0\right)\left(a_{i}, 0\right): i \in Z_{n-2}\right\} .
\end{aligned}
$$

Let $B_{r}=A_{r} \bigcup A_{r}^{\prime}$. Consider $B\left(L_{n}\left[C_{m}\right]\right)=B\left(C_{2 n}\left[C_{m}\right]\right) \cup\left(\bigcup_{r=1}^{n-2} B_{r}\right) \cup Q$, where $B\left(C_{2 n}\left[C_{m}\right]\right)$ is the $4-$ fold basis of $\mathcal{C}\left(C_{2 n}\left[C_{m}\right]\right)$ which is obtained in Theorem 3.7 of [11], so $B\left(C_{2 n}\left[C_{m}\right]\right)$ is linearly independent set of cycles in $\mathcal{C}\left(L_{n}\left[C_{m}\right]\right)$.

Let $B^{*}=B\left(C_{2 n}\left[C_{m}\right]\right) \cup Q$, the cycles of $Q$ enclose the finite faces of a planar ladder and form a basis to this ladder. Thus $Q$ is linearly independent set of cycles in $\mathcal{C}\left(L_{n}\left[C_{m}\right]\right)$. Moreover, $E(Q) \cap E\left(C_{2 n}\left[C_{m}\right]\right)$ induces a forest of paths, thus by Lemma 1.2 we conclude that $B^{*}$ is linearly independent.

For each $1 \leq r \leq n-2, A_{r}$ is Schemeichel's $4-$ fold basis of the subspace $\mathcal{C}\left(K_{\left(a_{r}, m\right),\left(b_{r}, m\right)}\right)$ that obtained in Theorem 2.4 of Schemeichel [18], thus each $A_{r}$ is linearly independent set of cycles in $\mathcal{C}\left(L_{n}\left[C_{m}\right]\right) . A_{r}^{\prime}$ is linearly independent set of cycles because the cycles of $A_{r}^{\prime}$ form the set of all finite faces of their corresponding planar subgraph that obtained by pasting them together successively with increasing $i$. Moreover, each cycle in $A_{r}^{\prime}$ contains one edge from the set

$$
\begin{aligned}
H= & \left\{\left(a_{i}, 0\right)\left(a_{i}, 1\right),\left(a_{i}, 1\right)\left(a_{i}, 2\right), \cdots,\left(a_{i}, m-1\right)\left(a_{i}, m\right)\right\} \\
& \cup\left\{\left(b_{i}, 0\right)\left(b_{i}, 1\right),\left(b_{i}, 1\right)\left(b_{i}, 2\right), \cdots,\left(b_{i}, m-1\right)\left(b_{i}, m\right)\right\},
\end{aligned}
$$

and this edge doesnot occur in any cycle of $A_{r}$, then the cycles of $A_{r}^{\prime}$ are linearly independent with the cycles of $A_{r}$, thus $B_{r}=A_{r} \cup A_{r}^{\prime}$ is linearly independent set of cycles in $\mathcal{C}\left(L_{n}\left[C_{m}\right]\right) . \bigcup_{r=1}^{n-2} B_{r}$ is linearly independent set of cycles in $\mathcal{C}\left(L_{n}\left[C_{m}\right]\right)$ because $E\left(B_{r}\right) \cap E\left(B_{k}\right)=\phi$, for all $1 \leq r, k \leq n-2$ and $r \neq k$. Moreover, $E\left(B^{*}\right) \cap E\left(\bigcup_{r=1}^{n-2} B_{r}\right)$ is a forest, thus by Lemma 1.2, $B\left(L_{n}\left[C_{m}\right]\right)=B^{*} \cup\left(\bigcup_{r=1}^{n-2} B_{r}\right)$ is a linearly independent set of cycles in $\mathcal{C}\left(L_{n}\left[C_{m}\right]\right)$. Since

$$
\begin{aligned}
\left|B\left(L_{n}\left[C_{m}\right]\right)\right| & =\left|B\left(C_{2 n}\left[C_{m}\right]\right)\right|+\left|\bigcup_{r=1}^{n-2} B_{r}\right|+|Q| \\
& =2 n m^{2}+1+\left(m^{2}-1\right)(n-2)+n-2 \\
& =3 n m^{2}-2 m^{2}+1=\operatorname{dim} \mathcal{C}\left(L_{n}\left[C_{m}\right]\right) .
\end{aligned}
$$

it follows that $B\left(L_{n}\left[C_{m}\right]\right)$ is a basis for $\mathcal{C}\left(L_{n}\left[C_{m}\right]\right)$. It is easy to verify that $B\left(L_{n}\left[C_{m}\right]\right)$ is a $4-$ fold basis for $\mathcal{C}\left(L_{n}\left[C_{m}\right]\right)$. Hence, $3 \leq \mathrm{b}\left(L_{n}\left[C_{m}\right]\right) \leq 4$ for all $n \geq 3$ and $m \geq 5$.

On the other hand, suppose that $\mathcal{C}\left(L_{n}\left[C_{m}\right]\right)$ has a $3-$ fold basis $\mathcal{B}$. Then, to prove that $\mathcal{B}$ can not exist for all $n \geq 3$ and $m \geq 6$, we have three cases:

Case 1. Suppose that $\mathcal{B}$ contains only 3 -cycles. Then $|\mathcal{B}| \leq 3 m(2 n)=6 n m$, since 
every 3 -cycles in $\mathcal{B}$ must contain an edge from the set

$$
S=\left\{\left(a_{i}, r\right)\left(a_{i}, r+1\right): r \in Z_{m}, i \in Z_{n}\right\} \cup\left\{\left(b_{i}, r\right)\left(b_{i}, r+1\right): r \in Z_{m}, i \in Z_{n}\right\},
$$

where $|S|=2 n m$, and the fold of every edge of $S$ is at most 3. But $|\mathcal{B}|=m^{2}(3 n-$ $2)+1=\operatorname{dim}\left(\mathcal{C}\left(L_{n}\left[C_{m}\right]\right)\right)$, so that $m^{2}(3 n-2)+1 \leq 6 n m$, and $m^{2}(3 n-2) \lesseqgtr$ $m^{2}(3 n-2)+1 \leq 6 n m$, since $m \geq 5$ then $5^{2}(3 n-2) \lesseqgtr 30 n$, which implies that $75 n-30 n-50 \lesseqgtr 0 \Leftrightarrow 45 n-50 \lesseqgtr 0$, which doesnot hold for all $n \geq 2$. Therefore, $m^{2}(3 n-2)+1 \leq 6 n m$ doesnot hold for all $n \geq 3$ and $m \geq 5$. Hence, $\mathcal{B}$ cannot be a basis of $\mathcal{C}\left(L_{n}\left[C_{m}\right]\right)$, a contradiction.

Case 2. Suppose that $\mathcal{B}$ consists only of cycles of length greater than or equal to 4 , then by Lemma 1.1 we have $4\left(m^{2}(3 n-2)+1\right) \leq 3\left(2 n m+m^{2}(3 n-2)\right)$ because $\operatorname{dim} \mathcal{C}\left(L_{n}\left[C_{m}\right]\right)=m^{2}(3 n-2)+1,\left|E\left(L_{n}\left[C_{m}\right]\right)\right|=m^{2}(3 n-2)+2 n m$, and $\left|C_{i}\right| \geq 4$ for every $C_{i} \in \mathcal{B}$. But, by Lemma 2.1, we have a contradiction for any $n \geq 3$ and $m \geq 6$.

Case 3. Suppose that $\mathcal{B}$ consists of $s 3$-cycles and $t$ cycles of length greater than or equal to 4 . Then $s \leq 3 m(2 n)$ because we have at most $3 m(2 n) 3$-cycles in $\mathcal{B}$ as we explained in Case 1 . Since $\left|E\left(L_{n}\left[C_{m}\right]\right)\right|=m^{2}(3 n-2)+2 n m$, and the fold of every edge of $L_{n}\left[C_{m}\right]$ is at most 3 in $\mathcal{B}$ and $3 \mathrm{~s}$ edges are joined to make the $s 3$-cycles, we have $t \leq\left[\frac{6 n m+9 n m^{2}-6 m^{2}-3 s}{4}\right]$. Then, $m^{2}(3 n-2)+$ $1=\operatorname{dim}\left(\mathcal{C}\left(L_{n}\left[C_{m}\right]\right)\right)=|\mathcal{B}|=s+t \leq s+\left[\frac{6 n m+9 n m^{2}-6 m^{2}-3 s}{4}\right]$, so that $m^{2}(3 n-2)+1 \leq s+\left[\frac{6 n m+9 n m^{2}-6 m^{2}-3 s}{4}\right]$. But, this inequality implies a contradiction for all $n \geq 3$ and $m \geq 6$ as proved in Lemma 2.2.

From the above three cases we deduce that $\mathcal{C}\left(L_{n}\left[C_{m}\right]\right)$ has no $3-$ fold basis for all $n \geq 3$ and $m \geq 6$. Hence, $\mathrm{b}\left(L_{n}\left[C_{m}\right]\right)=4$ for all $n \geq 3$ and $m \geq 6$.

Lemma 2.3 Let $m, n$ be two positive integers, $n \geq 3$, such that $4\left(3 n m^{2}-2 m^{2}-\right.$ $2 n+1) \leq 3\left(2 n m-2 n+3 n m^{2}-2 m^{2}\right)$, then this inequality holds for $m \lesseqgtr 4$.

Proof. Let $4\left(3 n m^{2}-2 m^{2}-2 n+1\right) \leq 3\left(2 n m-2 n+3 n m^{2}-2 m^{2}\right)$. We can simplify it to get the inequality $m^{2}(3 n-2) \leq 2 n(3 m+1)-4$. Dividing by $m(3 n-2)$ implies $m \leq \frac{2 n(3 m+1)-4}{m(3 n-2)} \leq 2\left(\frac{3 n}{3 n-2}\right)+\frac{2(n-2)}{m(3 n-2)}$. Since $\frac{3 n}{3 n-2}$ is a decreasing sequence for all $n \geq 3$ and $\frac{9}{7}$ is its maximum, we conclude that $m \lesseqgtr 4$.

Lemma 2.4 Let $m, n$ be two positive integers, $(n \geq 3)$, and $s \leq 3(2 n)(m-1)$, such that $m^{2}(3 n-2)-2 n+1 \leq s+\left[\frac{3 m^{2}(3 n-2)+6 n(m-1)-3 s}{4}\right]$, then $m \leqq 6$.

Proof. Since $\left[\frac{3 m^{2}(3 n-2)+6 n(m-1)-3 s}{4}\right] \leq \frac{3 m^{2}(3 n-2)+6 n(m-1)-3 s}{4}$, 
we have $m^{2}(3 n-2)-2 n+1 \leq s+\frac{3 m^{2}(3 n-2)+6 n(m-1)-3 s}{4}$. If we multiply by 4 , rearrange the terms and use the fact that $s \leq 6 n(m-1)$, we reach the inequality $m^{2}(3 n-2) \leq 12 n m-4(n-1)$. Dividing by $m(3 n-2)$ implies $m \leq$ $4 \frac{3 n}{3 n-2}-4 \frac{n-1}{m(3 n-2)} \varsubsetneqq 6$ being $\frac{3 n}{3 n-2}$ is a decreasing sequence for each $n \geq 3$ and $\frac{9}{7}$ is its maximum.

Corollary 2.1. For every $n \geq 3$ and $m \geq 5$, we have $3 \leq b\left(L_{n}\left[P_{m}\right]\right) \leq 4$. Moreover, if $n \geq 3$ and $m \geq 6$, then $b\left(L_{n}\left[P_{m}\right]\right)=4$.

Proof. The graph $L_{n}\left[P_{m}\right]$ is a subgraph of $L_{n}\left[C_{m}\right]$ consists of $3 n-2$ copies of $K_{m, m}$.

Let $B\left(L_{n}\left[P_{m}\right]\right)=B\left(L_{n}\left[C_{m}\right]\right)-M$, where

$$
\begin{aligned}
M= & \left\{\left(a_{i}, 0\right)\left(a_{i}, 1\right)\left(a_{i}, 2\right) \cdots\left(a_{i}, m-1\right)\left(a_{i}, 0\right): i \in Z_{n}\right\} \\
& \cup\left\{\left(b_{i}, 0\right)\left(b_{i}, 1\right)\left(b_{i}, 2\right) \cdots\left(b_{i}, m-1\right)\left(b_{i}, 0\right): i \in Z_{n}\right\} .
\end{aligned}
$$

and $B\left(L_{n}\left[C_{m}\right]\right)$ is the $4-$ fold basis of $\mathcal{C}\left(L_{n}\left[C_{m}\right]\right)$ that obtained in previous theorem. Because $B\left(L_{n}\left[C_{m}\right]\right)$ is linearly independent set and $B\left(L_{n}\left[P_{m}\right]\right) \subseteq B\left(L_{n}\left[C_{m}\right]\right)$, $B\left(L_{n}\left[P_{m}\right]\right)$ is linearly independent set of cycles in $\mathcal{C}\left(L_{n}\left[P_{m}\right]\right)$. Since $\left|B\left(L_{n}\left[P_{m}\right]\right)\right|=$ $3 n m^{2}-2 m^{2}+1-2 n=m^{2}(3 n-2)-2 n+1=\operatorname{dim} \mathcal{C}\left(L_{n}\left[P_{m}\right]\right)$ we have $B\left(L_{n}\left[P_{m}\right]\right)$ is a basis for $\mathcal{C}\left(L_{n}\left[P_{m}\right]\right)$. The fold of any edge of $L_{n}\left[P_{m}\right]$ in $B\left(L_{n}\left[P_{m}\right]\right)$ doesn't excess in $B\left(L_{n}\left[C_{m}\right]\right)$, thus $B\left(L_{n}\left[P_{m}\right]\right)$ is a $4-$ fold basis of $\mathcal{C}\left(L_{n}\left[P_{m}\right]\right)$. Hence, $3 \leq b\left(L_{n}\left[P_{m}\right]\right) \leq 4$.

On the other hand, suppose that $\mathcal{C}\left(L_{n}\left[P_{m}\right]\right)$ has a $3-$ fold basis $\mathcal{B}$, then to prove that such $\mathcal{B}$ cannot exist for all $n \geq 3$ and $m \geq 4$ we have three cases:

Case 1. Suppose that $\mathcal{B}$ consists only of 3-cycles, then $|\mathcal{B}| \leq 3(2 n)(m-1)=$ $6 n m-6 n$, because every 3 -cycle in $\mathcal{B}$ must contain an edge from the set $S=\left\{\left(a_{i}, r\right)\left(a_{i}, r+1\right): r \in Z_{m-1}, i \in Z_{n}\right\} \cup\left\{\left(b_{i}, r\right)\left(b_{i}, r+1\right): r \in Z_{m-1}, i \in Z_{n}\right\}$, and the fold of every edge is at most 3 . Since $|\mathcal{B}|=m^{2}(3 n-2)-2 n+1$, we have $m^{2}(3 n-2)-2 n+1 \leq 6 n m-6 n$. This inequality reduces to $m^{2}(3 n-2)+1 \leq 6 n m-4 n$. Dividing by $m(3 n-2)$ and simplifying this inequality gives $m+\frac{1}{m(3 n-2)} \leq$ $\frac{2}{3}\left(\frac{3 n}{3 n-2}\right)\left(\frac{3 m-2}{m}\right)<3$ because $\frac{3 n}{3 n-2}$ is a decreasing sequence for each $n \geq 3$ and $\frac{9}{7}$ is its maximum. Hence, $\mathcal{B}$ cannot be a basis of $\mathcal{C}\left(L_{n}\left[P_{m}\right]\right)$ for all $n \geq 3$ and $m \geq 4$, a contradiction.

Case 2. Suppose that $\mathcal{B}$ consists only of cycles of length greater than or equal to 4 , then by Lemma 1.1 we have $4\left(3 n m^{2}-2 m^{2}-2 n+1\right) \leq 3\left(2 n m-2 n+3 n m^{2}-2 m^{2}\right)$ because $\operatorname{dim} \mathcal{C}\left(L_{n}\left[P_{m}\right]\right)=3 n m^{2}-2 m^{2}-2 n+1,\left|E\left(L_{n}\left[P_{m}\right]\right)\right|=2 n m-2 n+3 n m^{2}$ $-2 m^{2}$, and $\left|C_{i}\right| \geq 4$, for every $C_{i} \in \mathcal{B}$. But by Lemma 2.3 , we have a contradiction for any $n \geq 3$ and $m \geq 6$. 
Case 3. Suppose that $\mathcal{B}$ consists of $s 3$-cycles and $t$ cycles of length greater than or equal to 4 , then $s \leq 3(m-1)(2 n)$, because we have at most $3(2 n)(m-1) 3$-cycles in $\mathcal{B}$ as we explained in Case 1. Since $\left|E\left(L_{n}\left[P_{m}\right]\right)\right|=m^{2}(3 n-2)+2 n(m-1)$, and the fold of every edge of $L_{n}\left[P_{m}\right]$ is at most 3 in $\mathcal{B}$ and $3 s$ edges are joined to make the $s 3$-cycles we have $t \leq\left[\frac{3\left(m^{2}(3 n-2)+2 n(m-1)\right)-3 s}{4}\right]$, then $m^{2}(3 n-2)-$ $2 n+1=\operatorname{dim} \mathcal{C}\left(L_{n}\left[P_{m}\right]\right)=|\mathcal{B}|=s+t \leq s+\left[\frac{3\left(m^{2}(3 n-2)+2 n(m-1)\right)-3 s}{4}\right]$ so that $m^{2}(3 n-2)-2 n+1 \leq s+\left[\frac{3\left(m^{2}(3 n-2)+2 n(m-1)\right)-3 s}{4}\right]$. But this inequality implies a contradiction for all $n \geq 3$ and $m \geq 6$ as proved in Lemma 2.4.

From the above three cases we deduce that $\mathcal{C}\left(L_{n}\left[P_{m}\right]\right)$ has no $3-$ fold basis for all $n \geq 3$ and $m \geq 6$. Hence, $\mathrm{b}\left(L_{n}\left[P_{m}\right]\right)=4$ for all $n \geq 3$ and $m \geq 6$.

Lemma 2.5. Let $m, n$ be two positive integers, $(n \geq 4)$, such that $4\left(3 n m^{2}+1\right) \leq$ $3\left(3 n m^{2}+2 n m\right)$. Then $m<2$.

Proof. Since $4\left(3 \mathrm{~nm}^{2}+1\right) \leq 3\left(3 \mathrm{~nm}^{2}+2 \mathrm{~nm}\right)$, we have $12 \mathrm{~nm} m^{2}+4 \leq 6 \mathrm{~nm}+9 \mathrm{~nm} m^{2}$. So that $3 \mathrm{~nm}^{2}+4 \leq 6 \mathrm{~nm}$, and $3 m+\frac{4}{n m} \leq 6$, this implies that $3 m<6$. Thus $m<2$.

Lemma 2.6 Let $m, n$ be two positive integers, $(n \geq 4), s \leq 6 n m$, such that $3 n m^{2}+1 \leq s+\left[\frac{6 n m+9 n m^{2}-3 s}{4}\right]$, then $m<4$.

Proof. Since $\left[\frac{6 n m+9 n m^{2}-3 s}{4}\right] \leq \frac{6 n m+9 n m^{2}-3 s}{4}$, we have $3 n m^{2}+1 \leq$ $s+\frac{6 n m+9 n m^{2}-3 s}{4}$. If we multiply by 4 rearrange the terms and use the fact that $s \leq 6 n m$, we get $m+\frac{4}{3 n m} \leq 4$, so that $m<4$.

Theorem 2.2. If $n \geq 4$ and $m \geq 5$, then $b\left(C L_{n}\left[C_{m}\right]\right)=4$.

Proof. The graph $C L_{n}\left[C_{m}\right]=L_{n}\left[C_{m}\right] \cup K_{\left(a_{n-1}, m\right),\left(a_{0, m}\right)} \cup K_{\left(b_{n-1}, m\right),\left(b_{0, m}\right)}$ consists of $3 n$ copies of the nonplanar graph $K_{m, m}$. It is clear that $C L_{n}\left[C_{m}\right]$ is a nonplanar graph because $L_{n}\left[C_{m}\right]$ is a nonplanar subgraph of it, thus by MacLane's Theorem $\mathrm{b}\left(C L_{n}\left[C_{m}\right]\right) \geq 3$.

To prove $b\left(C L_{n}\left[C_{m}\right]\right) \leq 4$, we exhibit a 4 -fold basis for $\mathcal{C}\left(C L_{n}\left[C_{m}\right]\right)$. Define the following sets of cycles in $\mathcal{C}\left(C L_{n}\left[C_{m}\right]\right)$ :

$$
\begin{aligned}
A_{1}= & \left\{\left(a_{n-1}, 0\right)\left(a_{0}, i\right)\left(a_{0}, i+1\right)\left(a_{n-1}, 0\right): 0 \leq i \leq m-2\right\} \\
& \cup\left\{\left(a_{0}, m-1\right)\left(a_{n-1}, i\right)\left(a_{n-1}, i+1\right)\left(a_{0}, m-1\right): 0 \leq i \leq m-2\right\}, \\
A_{2}= & \left\{\left(b_{n-1}, 0\right)\left(b_{0}, i\right)\left(b_{0}, i+1\right)\left(b_{n-1}, 0\right): 0 \leq i \leq m-2\right\} \\
& \cup\left\{\left(b_{0}, m-1\right)\left(b_{n-1}, i\right)\left(b_{n-1}, i+1\right)\left(b_{0}, m-1\right): 0 \leq i \leq m-2\right\},
\end{aligned}
$$




$$
\begin{aligned}
& A_{3}=B\left(K_{\left(a_{n-1, m}\right),\left(a_{0, m}\right)}\right), \\
& A_{4}=B\left(K_{\left(b_{n-1}, m\right),\left(b_{0, m}\right)}\right), \\
& Q_{1}=\left(a_{0}, m-1\right)\left(a_{1}, m-1\right)\left(a_{2}, m-1\right), \cdots,\left(a_{n-1}, m-1\right)\left(a_{0}, m-1\right), \\
& Q_{2}=\left(b_{0}, m-1\right)\left(b_{1}, m-1\right)\left(b_{2}, m-1\right), \cdots,\left(b_{n-1}, m-1\right)\left(b_{0}, m-1\right) .
\end{aligned}
$$

Let $B_{1}=A_{1} \cup A_{3}$ and $B_{2}=A_{2} \cup A_{4}$ and define $B\left(C L_{n}\left[C_{m}\right]\right)=B\left(L_{n}\left[C_{m}\right]\right)$ $\cup B_{1} \cup B_{2} \cup Q_{1} \cup Q_{2}$, where $B\left(L_{n}\left[C_{m}\right]\right)$ is the 4-fold basis of $\mathcal{C}\left(L_{n}\left[C_{m}\right]\right)$ that was exhibited in Theorem 2.1. So $B\left(L_{n}\left[C_{m}\right]\right)$ is linearly independent set of cycles in $\mathcal{C}\left(C L_{n}\left[C_{m}\right]\right)$. Since $Q_{1}$ contains the edge $\left(a_{n-1}, m-1\right)\left(a_{0}, m-1\right)$ and $Q_{2}$ contains the edge $\left(b_{n-1}, m-1\right)\left(b_{0}, m-1\right)$ and each of these edges doesn't occur in any cycle of $B\left(L_{n}\left[C_{m}\right]\right)$ then $B^{*}=B\left(L_{n}\left[C_{m}\right]\right) \cup Q_{1} \cup Q_{2}$ is linearly independent.

Note that $A_{3}$ and $A_{4}$ are Schemeichel's $4-$ fold bases of the subspaces $\mathcal{C}\left(K_{\left(a_{n-1, m}\right)}\right.$, $\left.\left(a_{0, m}\right)\right)$ and $\mathcal{C}\left(K_{\left(b_{n-1}, m\right),\left(b_{0}, m\right)}\right)$, respectively, and they were obtained in Theorem 2.4 of [18]. Thus, $A_{3}$ and $A_{4}$ are linearly independent set of cycles in $\mathcal{C}\left(C L_{n} C_{m}\right)$, $A_{3} \cap A_{4}=\phi$, so $A_{3} \cup A_{4}$ is linearly independent set of cycles. $A_{1}$ and $A_{2}$ are linearly independent sets of cycles because each of them represents the set of all finite faces of the corresponding planar graph that formed by pasting these cycles successively with increasing $i$, and $A_{1} \cap A_{2}=\phi$, so $A_{1} \cup A_{2}$ is linearly independent set of cycles. Since each linear combination of the cycles of $A_{1}$ contains at least one edge from the set

$$
\begin{aligned}
H_{1}= & \left\{\left(a_{0}, 0\right)\left(a_{0}, 1\right),\left(a_{0}, 1\right)\left(a_{0}, 2\right), \cdots,\left(a_{0}, m-1\right)\left(a_{0}, m\right)\right\} \\
& \cup\left\{\left(a_{n-1}, 0\right)\left(a_{n-1}, 1\right),\left(a_{n-1}, 1\right)\left(a_{n-1}, 2\right), \cdots,\left(a_{n-1}, m-1\right)\left(a_{n-1}, m\right)\right\}
\end{aligned}
$$

which doesnot appear in any cycle of $A_{3}$, then the cycles of $A_{1}$ are linearly independent with the cycles of $A_{3}$. Thus $B_{1}=A_{1} \cup A_{3}$ is a linearly independent set of cycles in $\mathcal{C}\left(C L_{n}\left[C_{m}\right]\right)$. Similarly, each linear combination of the cycles of $A_{2}$ contains at least one edge from the set

$$
\begin{aligned}
H_{2}= & \left\{\left(b_{0}, 0\right)\left(b_{0}, 1\right),\left(b_{0}, 1\right)\left(b_{0}, 2\right), \cdots,\left(b_{0}, m-1\right)\left(b_{0}, m\right)\right\} \\
& \cup\left\{\left(b_{n-1}, 0\right)\left(b_{n-1}, 1\right),\left(b_{n-1}, 1\right)\left(b_{n-1}, 2\right), \cdots,\left(b_{n-1}, m-1\right)\left(b_{n-1}, m\right)\right\}
\end{aligned}
$$

and this edge doesnot appear in any cycle of $A_{4}$, then the cycles of $A_{2}$ are linearly independent of the cycles of $A_{4}$. Thus $B_{2}=A_{2} \cup A_{4}$ is a linearly independent set of cycles in $\mathcal{C}\left(C L_{n}\left[C_{m}\right]\right)$. Since $E\left(B_{1}\right) \cap E\left(B_{2}\right)=\phi$, then $B_{1} \cup B_{2}$ is linearly independent. Moreover, $E\left(B_{1} \cup B_{2}\right) \cap E\left(B^{*}\right)$ is a forest, thus by Lemma 1.2, we conclude that $B\left(C L_{n}\left[C_{m}\right]\right)=B^{*} \cup B_{1} \cup B_{2}$ is linearly independent. Since

$$
\begin{aligned}
\left|B\left(C L_{n}\left[C_{m}\right]\right)\right| & =\left|B\left(L_{n}\left[C_{m}\right]\right)\right|+\left|B_{1}\right|+\left|B_{2}\right|+\left|Q_{1}\right|+\left|Q_{2}\right| \\
& =3 n m^{2}-2 m^{2}+1+m^{2}-1+m^{2}-1+1+1 \\
& =3 n m^{2}+1=\operatorname{dim} \mathcal{C}\left(C L_{n}\left[C_{m}\right]\right),
\end{aligned}
$$

it follows that $B\left(C L_{n}\left[C_{m}\right]\right)$ is a basis for $\mathcal{C}\left(C L_{n}\left[C_{m}\right]\right)$. It is easy to verify that $B\left(C L_{n}\left[C_{m}\right]\right)$ is a 4 -fold basis for $\mathcal{C}\left(C L_{n}\left[C_{m}\right]\right)$. Hence, b $\left(C L_{n}\left[C_{m}\right]\right)=4$ for all $n \geq 4$ and $m \geq 5$. 
On the other hand, suppose that $\mathcal{C}\left(C L_{n}\left[C_{m}\right]\right)$ has a $3-$ fold basis $\mathcal{B}$. Then we have three cases to prove that $\mathcal{B}$ can't exist for all $n \geq 4$ and $m \geq 5$ :

Case 1. Suppose that $\mathcal{B}$ contains only 3 -cycles then $|\mathcal{B}| \leq 3 m(2 n)=6 n m$, because every 3 - cycles in $\mathcal{B}$ must contain an edge from the set

$$
\begin{aligned}
S= & \left\{\left(a_{i}, 0\right)\left(a_{i}, 1\right),\left(a_{i}, 1\right)\left(a_{i}, 2\right), \cdots,\left(a_{i}, m-1\right)\left(a_{i}, 0\right): i \in Z_{n}\right\} \\
& \cup\left\{\left(b_{i}, 0\right)\left(b_{i}, 1\right),\left(b_{i}, 1\right)\left(b_{i}, 2\right), \cdots,\left(b_{i}, m-1\right)\left(b_{i}, 0\right): i \in Z_{n}\right\},
\end{aligned}
$$

and the fold of every edge of $S$ is at most 3 . But $|\mathcal{B}|=3 \mathrm{~nm}^{2}+1$, so that $3 \mathrm{~nm}^{2}+1 \leq$ $3 m(2 n)=6 n m$, but this inequality doesnot hold for all $n \geq 2$ and $m \geq 2$. Hence, $\mathcal{B}$ cannot be a basis of $\mathcal{C}\left(C L_{n}\left[C_{m}\right]\right)$ for all $n \geq 4$ and $m \geq 5$, a contradiction.

Case 2. Suppose that $\mathcal{B}$ consists only of cycles of length greater than or equal to 4 then by Lemma 1.1 we have $4\left(3 \mathrm{~nm}^{2}+1\right) \leq 3\left(3 \mathrm{~nm}^{2}+2 \mathrm{~nm}\right)$, because $\operatorname{dim}$ $\mathcal{C}\left(C L_{n}\left[C_{m}\right]\right)=3 n m^{2}+1,\left|E\left(L_{n}\left[C_{m}\right]\right)\right|=3 n m^{2}+2 n m$, and $\left|C_{i}\right| \geq 4$ for every $C_{i} \in \mathcal{B}$. But by Lemma 2.5, we have a contradiction for any $n \geq 4$ and $m \geq 5$.

Case 3. Suppose that $\mathcal{B}$ consists of $s 3-$ cycles and $t$ cycles of length greater than or equal to 4 , then $s \leq 3 m(2 n)$, because we have at most $3 m(2 n) 3$-cycles in $\mathcal{B}$ as we explained in Case 1 . Since $\left|E\left(L_{n}\left[C_{m}\right]\right)\right|=3 \mathrm{~nm}^{2}+2 n m$, and the fold of every edge of $C L_{n}\left[C_{m}\right]$ is at most 3 in $\mathcal{B}$ and $3 s$ edges are joined to make the $s 3-$ cycles, we have $t \leq\left[\frac{6 n m+9 n m^{2}-3 s}{4}\right]$. Then $3 n m^{2}+1=\operatorname{dim} \mathcal{C}\left(C L_{n}\left[C_{m}\right]\right)=|\mathcal{B}|=$ $s+t \leq s+\left[\frac{6 n m+9 n m^{2}-3 s}{4}\right]$. Thus $3 n m^{2}+1 \leq s+\left[\frac{6 n m+9 n m^{2}-3 s}{4}\right]$. But this inequality implies a contradiction for all $n \geq 4$ and $m \geq 5$, as it was proved in Lemma 2.6.

Lemma 2.7. Let $m, n$ be two positive integers, $(n \geq 4)$, such that $4\left(3 n m^{2}-2 n+\right.$ $1) \leq 3\left(3 n m^{2}+2 n m-2 n\right)$. Then $m \lesseqgtr 3$.

Proof. Since $4\left(3 n m^{2}-2 n+1\right) \leq 3\left(3 n m^{2}+2 n m-2 n\right)$ we have $12 n m^{2}-8 n+4 \leq$ $9 n m^{2}+6 n m-6 n$, then $3 n m^{2}+4-2 n \leq 6 n m$, thus $m \leq 2+\left(\frac{2}{3 m}-\frac{4}{3 n m}\right)$. Therefore, $m \lesseqgtr 3$.

Lemma 2.8. Let $m, n$ be two positive integers, $(n \geq 4), s \leq 6 n m-6 n$ such that $3 n m^{2}-2 n+1 \leq s+\left[\frac{6 n m+9 n m^{2}-6 n-3 s}{4}\right]$. Then $m \leqq 5$.

Proof. Since $\left[\frac{6 n m+9 n m^{2}-6 n-3 s}{4}\right] \leqq \frac{6 n m+9 n m^{2}-6 n-3 s}{4}$ we get $3 n m^{2}-$ $2 n+1 \leq s+\frac{6 n m+9 n m^{2}-6 n-3 s}{4}$. If we multiply by 4 and rearrange the terms and use the fact that $s \leq 6 n m-6 n$ we get the inequality $3 n m^{2} \leq 2 n+12 n m-4$. Dividing by $3 n m$ implies $m \leq 4+\left(\frac{2}{3 m}-\frac{4}{3 n m}\right)=4+\frac{2 n-4}{3 n m}$. since $n \geq 4$ this 
inequality holds only for $m \lesseqgtr 5$.

Corollary 2.2 For every $n \geq 4$ and $m \geq 5, b\left(C L_{n}\left[P_{m}\right]\right)=4$.

Proof. The graph $C L_{n}\left[P_{m}\right]$ is a subgraph of $C L_{n}\left[C_{m}\right]$ that consists of $3 n$ copies of $K_{m, m}$.

Let $B\left(C L_{n}\left[P_{m}\right]\right)=B\left(L_{n}\left[C_{m}\right]\right)-M$, where

$$
\begin{aligned}
M= & \left\{\left(a_{i}, 0\right)\left(a_{i}, 1\right)\left(a_{i}, 2\right) \cdots\left(a_{i}, m-1\right)\left(a_{i}, 0\right): i \in Z_{n}\right\} \\
& \cup\left\{\left(b_{i}, 0\right)\left(b_{i}, 1\right)\left(b_{i}, 2\right) \cdots\left(b_{i}, m-1\right)\left(b_{i}, 0\right): i \in Z_{n}\right\}
\end{aligned}
$$

and $B\left(C L_{n}\left[C_{m}\right]\right)$ is the $4-$ fold basis of $\mathcal{C}\left(C L_{n}\left[C_{m}\right]\right)$ that was exhibited in Theorem 2.1. Since $B\left(C L_{n}\left[C_{m}\right]\right)$ is linearly independent set and $B\left(C L_{n}\left[P_{m}\right]\right) \subseteq$ $B\left(C L_{n}\left[C_{m}\right]\right)$, then $\mathrm{B}\left(C L_{n}\left[P_{m}\right]\right)$ is linearly independent set of cycles in $\mathcal{C}\left(C L_{n}\left[P_{m}\right]\right)$. Since $\left|B\left(C L_{n}\left[P_{m}\right]\right)\right|=3 n m^{2}+1-2 n=\operatorname{dim} \mathcal{C}\left(C L_{n}\left[P_{m}\right]\right)$, we conclude that $B\left(C L_{n}\left[P_{m}\right]\right)$ is a basis for $\mathcal{C}\left(C L_{n}\left[P_{m}\right]\right)$. The fold of any edge of $C L_{n}\left[P_{m}\right]$ in $B\left(C L_{n}\left[P_{m}\right]\right)$ is at most as it is in $B\left(C L_{n}\left[C_{m}\right]\right)$. Thus $B\left(C L_{n}\left[P_{m}\right]\right)$ is 4 -fold basis of $\mathcal{C}\left(C L_{n}\left[P_{m}\right]\right)$. Hence, $\mathrm{b}\left(C L_{n}\left[P_{m}\right]\right)=4$ for each $n \geq 4$ and $m \geq 5$.

On the other hand, suppose that $\mathcal{C}\left(C L_{n}\left[P_{m}\right]\right)$ has a $3-$ fold basis $\mathcal{B}$, then we have the following three cases to prove that $\operatorname{such} \mathcal{B}$ doesnot exist for all $n \geq 4$ and $m \geq 5$ :

Case1. Suppose that $\mathcal{B}$ contains only 3 -cycles, then $|\mathcal{B}| \leq 3(m-1)(2 n)=6 n m-6 n$, because every 3 -cycles in $\mathcal{B}$ must contain an edge from the set

$$
\begin{aligned}
S= & \left\{\left(a_{i}, 0\right)\left(a_{i}, 1\right),\left(a_{i}, 1\right)\left(a_{i}, 2\right), \cdots,\left(a_{i}, m-2\right)\left(a_{i}, m-1\right): i \in Z_{n}\right\} \\
& \cup\left\{\left(b_{i}, 0\right)\left(b_{i}, 1\right),\left(b_{i}, 1\right)\left(b_{i}, 2\right), \cdots,\left(b_{i}, m-2\right)\left(b_{i}, m-1\right): i \in Z_{n}\right\},
\end{aligned}
$$

and the fold of every edge of $S$ is at most 3 . But $|\mathcal{B}|=\operatorname{dim} \mathcal{C}\left(C L_{n}\left[P_{m}\right]\right)=3 \mathrm{~nm}^{2}$ $-2 n+1$, so $3 n m^{2}-2 n+1 \leq 6 n m-6 n$. Then $3 n m^{2}+1 \leq 6 n m-4 n$. If we divide by $3 n m$ we get $m+\frac{1}{3 n m}+\frac{4}{3 m} \leq 2$, which implies $m \leqq 1$. Hence $\mathcal{B}$ cannot be a basis of $\mathcal{C}\left(C L_{n}\left[P_{m}\right]\right)$ for all $n \geq 4$ and $m \geq 5$, a contradiction.

Case 2. Suppose that $\mathcal{B}$ consists only of cycles of length greater than or equal to 4 , then by Lemma 1.1 we have $4\left(3 n m^{2}-2 n+1\right) \leq 3\left(3 n m^{2}+2 n m-2 n\right)$, because $\operatorname{dim} \mathcal{C}\left(C L_{n}\left[P_{m}\right]\right)=3 n m^{2}-2 n+1,\left|E\left(L_{n}\left[P_{m}\right]\right)\right|=3 n m^{2}+2 n m-2 n$, and $\left|C_{i}\right| \geq$ 4 for every $C_{i} \in \mathcal{B}$. But by Lemma 2.7 we have a contradiction for any $n \geq 4$ and $m \geq 5$.

Case 3. Suppose that $\mathcal{B}$ consists of $s 3-$ cycles and $t$ cycles of length greater than or equal to 4 , then $s \leq 3(m-1)(2 n)$, because we have at most $3(2 n)(\mathrm{m}-1)$ $3-$ cycles in $\mathcal{B}$ as we explained in Case 1 . Since $\left|E\left(L_{n}\left[P_{m}\right]\right)\right|=3 n m^{2}+2 n m-2 n$, and the fold of every edge of $C L_{n}\left[P_{m}\right]$ is at most 3 in $\mathcal{B}$ and $3 s$ edges are joined to make the $s 3-$ cycles, we have $t \leq\left[\frac{6 n m+9 n m^{2}-6 n-3 s}{4}\right]$. Then $3 n m^{2}-2 n+1=\operatorname{dim} \mathcal{C}\left(C L_{n}\left[P_{m}\right]\right)=|\mathcal{B}|=s+t \leq s+\left[\frac{6 n m+9 n m^{2}-6 n-3 s}{4}\right]$. 
So that $3 n m^{2}-2 n+1 \leq s+\left[\frac{6 n m+9 n m^{2}-6 n-3}{4}\right]$. But, this inequality implies a contradiction for all $n \geq 4$ and $m \geq 5$ as we proved in Lemma 2.8.

Theorem 2.3. For every $n \geq 4$ and $m \geq 5, b\left(M L_{n}\left[C_{m}\right]\right)=4$.

Proof. The graph $M L_{n}\left[C_{m}\right]=L_{n}\left[C_{m}\right] \cup K_{\left(a_{n-1}, m\right),\left(b_{0, m}\right)} \cup K_{\left(b_{n-1}, m\right),\left(a_{0, m}\right)}$ consists of $3 n$ copies of the nonplanar graph $K_{m, m}$. It is clear that $M L_{n}\left[C_{m}\right]$ is a nonplanar graph because $L_{n}\left[C_{m}\right]$ is a nonplanar subgraph of it, thus by Maclane's theorem $b\left(M L_{n}\left[C_{m}\right]\right) \geq 3$.

To prove $\mathrm{b}\left(M L_{n}\left[C_{m}\right]\right) \leq 4$, we exhibit a $4-$ fold basis for $\mathcal{C}\left(M L_{n}\left[C_{m}\right]\right)$. Define the following sets of cycles in $\mathcal{C}\left(M L_{n}\left[C_{m}\right]\right)$ :

$$
\begin{aligned}
A_{1}= & \left\{\left(a_{n-1}, 0\right)\left(b_{0}, i\right)\left(b_{0}, i+1\right)\left(a_{n-1}, 0\right): 0 \leq i \leq m-2\right\} \\
& \cup\left\{\left(b_{0}, m-1\right)\left(a_{n-1}, i\right)\left(a_{n-1}, i+1\right)\left(b_{0}, m-1\right): 0 \leq i \leq m-2\right\}, \\
A_{2}= & \left\{\left(b_{n-1}, 0\right)\left(a_{0}, i\right)\left(a_{0}, i+1\right)\left(b_{n-1}, 0\right): 0 \leq i \leq m-2\right\} \\
& \cup\left\{\left(a_{0}, m-1\right)\left(b_{n-1}, i\right)\left(b_{n-1}, i+1\right)\left(a_{0}, m-1\right): 0 \leq i \leq m-2\right\}, \\
A_{3}= & B\left(K_{\left(a_{n-1}, m\right),\left(b_{0, m}\right)}\right) \\
A_{4}= & B\left(K_{\left(b_{n-1, m}\right),\left(a_{0, m}\right)}\right) \\
Q_{1}= & \left(a_{0}, m-1\right)\left(a_{1}, m-1\right), \cdots,\left(a_{n-1}, m-1\right)\left(b_{0}, m-1\right)\left(a_{0}, m-1\right), \\
Q_{2}= & \left(b_{0}, m-1\right)\left(b_{1}, m-1\right)\left(b_{2}, m-1\right), \cdots,\left(b_{n-1}, m-1\right)\left(a_{0}, m-1\right)\left(b_{0}, m-1\right) .
\end{aligned}
$$

Let $B_{1}=A_{1} \cup A_{3}, B_{2}=A_{2} \cup A_{4}$. Define $B\left(M L_{n}\left[C_{m}\right]\right)=B\left(L_{n}\left[C_{m}\right]\right) \cup$ $B_{1} \cup B_{2} \cup Q_{1} \cup Q_{2}$, where $B\left(L_{n}\left[C_{m}\right]\right)$ is the 4 -fold basis of $\mathcal{C}\left(L_{n}\left[C_{m}\right]\right)$ that was exhibited in Theorem 2.1. So $B\left(L_{n}\left[C_{m}\right]\right)$ is linearly independent set of cycles in $\mathcal{C}\left(M L_{n}\left[C_{m}\right]\right)$. Since $Q_{1}$ contains the edge $\left(a_{n-1}, m-1\right)\left(b_{0}, m-1\right)$ and $Q_{2}$ contains the edge $\left(b_{n-1}, m-1\right)\left(a_{0}, m-1\right)$ and each of these edges doesn't occur in any cycle of $B\left(L_{n}\left[C_{m}\right]\right)$ then $B^{*}=B\left(L_{n}\left[C_{m}\right]\right) \cup Q_{1} \cup Q_{2}$ is linearly independent.

Note that $A_{3}$ and $A_{4}$ are Schemeichel's $4-$ fold bases of the subspaces $\mathcal{C}\left(K_{\left(a_{n-1, m}\right)}\right.$, $\left.\left(b_{0, m}\right)\right)$ and $\mathcal{C}\left(K_{\left(b_{n-1}, m\right),\left(a_{0}, m\right)}\right)$, respectively, and they were obtained in Theorem 2.4 of [18]. Thus $A_{3}$ and $A_{4}$ are linearly independent set of cycles in $\mathcal{C}\left(M L_{n}\left[C_{m}\right]\right)$, and $A_{3} \cap A_{4}=\phi$, so $A_{3} \cup A_{4}$ is linearly independent sets of cycles. $A_{1}$ and $A_{2}$ are linearly independent sets of cycles because each of them represents the set of all finite faces of the corresponding planar graph that formed by pasting these cycles successively with increasing $i$, and $A_{1} \cap A_{2}=\phi$, so $A_{1} \cup A_{2}$ is linearly independent set of cycles. Since each linear combination of cycles of $A_{1}$ contains at least one edge from the set

$$
\begin{aligned}
H_{1}= & \left\{\left(b_{0}, 0\right)\left(b_{0}, 1\right),\left(b_{0}, 1\right)\left(b_{0}, 2\right), \cdots,\left(b_{0}, m-1\right)\left(b_{0}, m\right)\right\} \\
& \cup\left\{\left(a_{n-1}, 0\right)\left(a_{n-1}, 1\right),\left(a_{n-1}, 1\right)\left(a_{n-1}, 2\right), \cdots,\left(a_{n-1}, m-1\right)\left(a_{n-1}, m\right)\right\}
\end{aligned}
$$

and this edge doesnot appear in any cycle of $A_{3}$ then the cycles of $A_{1}$ are linearly independent of the cycles of $A_{3}$. Thus $B_{1}=A_{1} \cup A_{3}$ is linearly independent set of cycles in $\mathcal{C}\left(C L_{n}\left[C_{m}\right]\right)$. Similarly, each linear combination of cycles of $A_{2}$ contains 
at least one edge from the set

$$
\begin{aligned}
H_{2}= & \left\{\left(a_{0}, 0\right)\left(a_{0}, 1\right),\left(a_{0}, 1\right)\left(a_{0}, 2\right), \cdots,\left(a_{0}, m-1\right)\left(a_{0}, m\right)\right\} \\
& \cup\left\{\left(b_{n-1}, 0\right)\left(b_{n-1}, 1\right),\left(b_{n-1}, 1\right)\left(b_{n-1}, 2\right), \cdots,\left(b_{n-1}, m-1\right)\left(b_{n-1}, m\right)\right\},
\end{aligned}
$$

and this edge doesnot appear in any cycle of $A_{4}$ then the cycles of $A_{2}$ are linearly independent of the cycles of $A_{4}$, thus $B_{2}=A_{2} \cup A_{4}$ is linearly independent set of cycles in $\mathcal{C}\left(M L_{n}\left[C_{m}\right]\right)$. Since $E\left(B_{1}\right) \cap E\left(B_{2}\right)=\phi$ the set $B_{1} \cup B_{2}$ is linearly independent. Moreover, $E\left(B_{1} \cup B_{2}\right) \cap E\left(B^{*}\right)$ is a forest, then by Lemma 1.2 we conclude that $B\left(M L_{n}\left[C_{m}\right]\right)=B^{*} \cup B_{1} \cup B_{2}$ is linearly independent set of cycles. Since

$$
\begin{aligned}
\left|B\left(M L_{n}\left[C_{m}\right]\right)\right| & =\left|B\left(L_{n}\left[C_{m}\right]\right)\right|+\left|B_{1}\right|+\left|B_{2}\right|+\left|Q_{1}\right|+\left|Q_{2}\right| \\
& =3 n m^{2}-2 m^{2}+1+\left(m^{2}-1\right)+\left(m^{2}-1\right)+1+1 \\
& =3 n m^{2}+1=\operatorname{dim} \mathcal{C}\left(M L_{n}\left[C_{m}\right]\right),
\end{aligned}
$$

it follows that $B\left(M L_{n}\left[C_{m}\right]\right)$ is a basis for $\mathcal{C}\left(M L_{n}\left[C_{m}\right]\right)$. It is easy to see that $B\left(M L_{n}\left[C_{m}\right]\right)$ is a $4-$ fold basis for $\mathcal{C}\left(M L_{n}\left[C_{m}\right]\right)$. Hence, $b\left(M L_{n}\left[C_{m}\right]\right)=4$ for all $n \geq 4$ and $m \geq 5$.

On the other hand, suppose that $\mathcal{C}\left(M L_{n}\left[C_{m}\right]\right)$ has a $3-$ fold basis $\mathcal{B}$. Then we have three cases to prove that $\mathcal{B}$ can't exist for all $n \geq 4$ and $m \geq 5$. They are identical to the three cases of Theorem 2.2, which we omit here.

Corollary 2.3. For every $n \geq 4$ and $m \geq 5,\left(b M L_{n}\left[P_{m}\right]\right)=4$.

Proof. The graph $M L_{n}\left[P_{m}\right]$ is a subgraph of $M L_{n}\left[C_{m}\right]$ that consists of $3 n$ copies of $K_{m, m}$.

Let $B\left(M L_{n}\left[P_{m}\right]\right)=B\left(M L_{n}\left[C_{m}\right]\right)-M$, where

$$
\begin{aligned}
M= & \left\{\left(a_{i}, 0\right)\left(a_{i}, 1\right),\left(a_{i}, 1\right)\left(a_{i}, 2\right), \cdots,\left(a_{i}, m-1\right)\left(a_{i}, 0\right): i \in Z_{n}\right\} \\
& \cup\left\{\left(b_{i}, 0\right)\left(b_{i}, 1\right),\left(b_{i}, 1\right)\left(b_{i}, 2\right), \cdots,\left(b_{i}, m-1\right)\left(b_{i}, 0\right): i \in Z_{n}\right\},
\end{aligned}
$$

and $B\left(M L_{n}\left[C_{m}\right]\right)$ is the $4-$ fold basis of $\mathcal{C}\left(M L_{n}\left[C_{m}\right]\right)$ that was exhibited in Theorem 2.3. Since $B\left(M L_{n}\left[C_{m}\right]\right)$ is linearly independent set and $B\left(M L_{n}\left[P_{m}\right]\right.$ $\subseteq B\left(M L_{n}\left[C_{m}\right]\right)$, then $B\left(M L_{n}\left[P_{m}\right]\right)$ is linearly independent set of cycles in $\mathcal{C}\left(M L_{n}\left[P_{m}\right]\right)$. Since $\left|B\left(M L_{n}\left[P_{m}\right]\right)\right|=3 n m^{2}-2 n+1=\operatorname{dim} \mathcal{C}\left(M L_{n}\left[P_{m}\right]\right)$, we conclude that $B\left(M L_{n}\left[P_{m}\right]\right)$ is a basis for $\mathcal{C}\left(M L_{n}\left[P_{m}\right]\right)$. The fold of any edge of $M L_{n}\left[P_{m}\right]$ in $B\left(M L_{n}\left[P_{m}\right]\right)$ is the same as it is in $B\left(M L_{n}\left[C_{m}\right]\right)$, thus $B\left(M L_{n}\left[P_{m}\right]\right)$ is a 4 -fold basis of $\mathcal{C}\left(M L_{n}\left[P_{m}\right]\right)$. Hence, $\mathrm{b}\left(M L_{n}\left[P_{m}\right]\right)=4$ for each $n \geq 4$ and $m \geq 5$.

On the other hand, suppose that $\mathcal{C}\left(M L_{n}\left[P_{m}\right]\right)$ has a 3 -fold basis $\mathcal{B}$. Then we have three cases to prove that $\mathcal{B}$ can't exist for all $n \geq 4$ and $m \geq 5$. They are identical to the three cases of Theorem 2.2, which we omit here. 


\section{References}

[1] A. A. Ali, The basis number of the direct product of paths and cycles, Ars. Combin., 27(1989), 155-163.

[2] A. A. Ali, The basis number of complete multipartite graphs, Ars. combin., 28(1989), 41-49.

[3] A. A. Ali and G. T. Marougi, The basis number of the strong product of graphs, Mutah Lil-Buhoth wa Al-Dirasat, 7(1)(1992), 211-222.

[4] A. A. Ali and G. T. Marougi, The basis number of the Lexicographic product of graphs, Ars. combin., 36(1993), 271-282.

[5] A. S. Alsardary and J. Wojciechowski, The basis number of the powers of the complete graph, Discrete Math., 188(1-3)(1998), 13-25.

[6] M. Y. Alzoubi, On the basis number of graphs, M. Sc thesis Yarmouk university, (1992).

[7] M. Y. Alzoubi and M. M. M. Jaradat, The basis number of the composition of theta graphs with stars and wheels, Acta Math. Hungar., 103(4)(2004), 201-209.

[8] M. Y. Alzoubi and M. M. M. Jaradat, On the Basis Number of the Composition of Different Ladders with Some Graphs, I. J. M. M. S.,2005(12)(2005), 1861-1868.

[9] J. A. Banks and E. F. Schemeichel, The basis number of n-Cube, J. Combin. Theory ser. B, 33(2)(1982), 95-100.

[10] Bondy, J. A. and Murty, U. S. R.. Graph Theory with Applications, America Elsevier Publishing Co. Inc., New York, 1976.

[11] M. Q. Hailat and M. Y. Alzoubi, The basis number of the composition of graph, Istnbul Univ. Fen Fak. Mat. Der., 53(1994), 43-60.

[12] M. M. Jaradat, On the basis number of the direct product of graphs, Aust. J.Cobin., 27(2003), 293-306.

[13] M. M. M. Jaradat, M. Y. Alzoubi and E. A. Rawashdeh, The basis number of the Lexicographic product of different ladders, SUT Journal of Mathematics, 40(2)(2004), 91-101.

[14] M. M. Jaradat, The basis number of the direct product of a theta graph and a path, Ars. Combin., 75(2005), 105-111.

[15] M. M. M. Jaradat, An upper bound of the basis number of the strong product of graphs, Discussion Mathematicea Graph Theory, 25(3)(2005), 391-406.

[16] Jonathan Gross and Jay Yellen, Graph Theory and Its Applications, CRC Press New York, 1999.

[17] S. F. MacLane, A combinatorial condition for planar graphs, Fundamenta Math., 28(1937), 22-32.

[18] E. F. Schemeichel, The basis number of graphs, J. Combin. Theory ser. B, 30(2)(1981), 123-129. 